\title{
A Systematic Spectral-Tau Method for the Solution of Fuzzy Fractional Diffusion and Fuzzy Fractional Wave Equations
}

\author{
Najeeb Alam Khan, Oyoon Abdul Razzaq \\ Department of Mathematical Sciences, University of Karachi, Karachi 75270, Pakistan \\ E-mail: njbalam@yahoo.com
}

\begin{abstract}
In this paper, fuzzy fractional diffusion equations (FFDEs) and fuzzy fractional wave equations (FFWEs), subjected to initial and boundary conditions are considered. As these equations have significant applications in physics and engineering, a methodical spectral-tau scheme is utilized to obtain efficient solutions of FFDE and FFWE. For this purpose, shifted Chebyshev polynomials (SCPs) together with its operational matrix of integration in Riemann-Liouville sense and operation matrix of derivative in Caputo sense are employed to approximate the fuzzy-valued functions, their integral and differential terms, respectively. The proposed method is applied to some illustrative examples considered under generalized Hukuhara partial differentiability ( $g H_{P}$-differentiability). Graphical results are included with error bar plots of each example that show the efficiency and convergence of the method towards the exact solution.
\end{abstract}

2010 Mathematics Subject Classification. 35L05. 35K05, 34A07

Keywords. Fuzzy-valued functions, Generalized Hukuhara differentiability, shifted Chebyshev polynomials, operational matrix.

\section{Introduction}

Fuzzy ordinary differential equations of integer and fractional order have achieved immense prosperity and popularity in different fields of science and engineering, for the reason of having the property of considering all the uncertain parameters which are usually kept constant in simple differential models. Its enormous applications have rapidly increased the practice of innovating new numerical and analytical methods among researchers so that the solutions of the problems can be obtained more accurately with less computation and within minimum time $[1,2,3,4]$. On contrary, fuzzy partial differential equations (FPDE) have got less attention than the fuzzy ordinary differential equations in this regard. In literature, very few papers exist on fuzzy diffusion and wave like equations of integer as well as fractional order. For instance, Buckley et al. [5] introduced the elementary of fuzzy partial differential equations, Behzadi [6] considered Cauchy reaction diffusion equation with fuzzy initial conditions and used Picard method to discuss the existence and uniqueness of the solution, fuzzy fractional heat equation is found in paper of Ghazanfari et al. [7] where differential transformation method is applied to attain the solution. Allahviranloo et al. described fuzzy heat and wave equations using difference methods [8], introduced generalized Hukuhara partial derivative for fuzzy heat equation and obtained the solutions [9] and also determined different solutions of fuzzy wave-like equations using variational iteration method [10].

Spectral methods are widely used tools that are measured to be very efficient for investigating solutions of ordinary and partial differential equations, function approximation and variational problems in science and engineering. Tau method can be considered as one of the common and frequently used spectral scheme utilized for numerical simulation of partial and ordinary differential 
equations of integer and fractional order. This method employs orthogonal polynomials as the basis functions to approximate the global functions smoothly. In recent years due to its high accurate approximations, it has gained considerable significance for solving differential equations in bounded as well as unbounded domains. Mostly, for bounded domain differential equations the orthogonal polynomials Legendre and Chebyshev are used for the approximations, for this instance see $[11,12,13,14,15,16]$, whereas Hermite and Laguerre are appropriately chosen to approximate solutions on the infinite and semi infinite domains, respectively, for instance see $[17,18,19,20]$ and many others. In spectral tau method the problem is completely discretized with the help of the operational matrix of integration and derivative of the respective orthogonal polynomials. In this connection, many authors $[21,22,23,24]$, have derived the operational matrix of derivative and integration of different orthogonal polynomials and have applied it on different problems.

The ongoing applications of spectral tau method inspired to utilize it on scrutinizing solutions of FPDEs more accurately. Hence this paper assesses solutions of fuzzy fractional diffusion equation (FFDE) and fuzzy fractional wave equation (FFWE), which have not been considered in literature hitherto. Since the problems under consideration are in finite domain, therefore shifted Chebyshev polynomials (SCPs) (see e.g. [23] and the references therein) are employed as basic orthogonal functions together with its operational matrix of fractional integration and Caputo derivative. The ability of this method, in converting the differential equation into a completely fuzzy algebraic system of equations [?, 25], greatly simplifies the problem and makes it easy to analyze the solutions more rapidly. Here after converting the differential equation to fully integrated problem, the fuzzy-valued function and its integrals are approximated using truncated series of SCPs and its operational matrices. Moreover, the rest of the manuscript is arranged as follows: next section contains some preliminary definitions and notations of fuzzy set theory and fuzzy fractional calculus that are assumed to be essential for the remaining paper. Section 3 encompasses the explanation of SCPs, shifted Chebyshev operational matrix (SCOM) of Caputo fractional derivative and SCOM of Riemann-Liouville fractional integration. In Section 4, approximations of univariate and bivariate fuzzy-valued functions using truncated series of SCPs are described. The methodology of the proposed scheme is elucidated systematically in Section 5. Section 6 comprises some illustrative examples of FFDE and FFWE. Additionally, discussion on obtained results in Section 6 and an effective conclusion is drawn in Section 7.

\section{Preliminaries}

This section consists of brief descriptions of all prerequisites of this paper. We define some definitions and notions of fuzzy set theory [26] followed by the fuzzy Riemann-Liouville fractional integral (FRLFI) and fuzzy Caputo gH-fractional derivative (FCgHFD) (see e.g. [27, 28] and the references therein).

\subsection{Fuzzy Set Theory}

Let $\Lambda_{F}$ be the set of subsets of the real line defined as

$$
\Lambda_{F}=\{\kappa \mid \kappa: \Re \rightarrow[0,1]\}
$$

Then $\Lambda_{F}$ is said to be set of fuzzy numbers $\kappa$, where $\kappa$ is normal, fuzzy convex, upper semi continuous 
and compactly supported on $\Re$. Each $\kappa$ can be represented in $\wp$-level by nonempty compact intervals as $[\kappa]^{\wp}=[\underline{\kappa}(\wp), \bar{\kappa}(\wp)]$ for $\wp \in[0,1]$, where $[\kappa]^{\wp}$ is said to be $\wp$-level set of $\kappa$ with $\underline{\kappa}(\wp)$ and $\bar{\kappa}(\wp)$, its nondecreasing lower function and nonincreasing upper function, respectively, and are bounded left continuous on $(0,1]$ and right continuous at $\wp=0$. The length of the level set $[\kappa]^{\wp}$ is defined as $\ell\left([\kappa]^{\wp}\right)=\bar{\kappa}(\wp)-\underline{\kappa}(\wp)$. Addition and multiplication of any arbitrary $\kappa, \varpi \in \Lambda_{F}$ is identified as, for $\wp \in[0,1]$ and $c \in \Re$

$$
\begin{aligned}
& (a)[\kappa \oplus \varpi]^{\wp}=[\underline{\kappa}(\wp)+\underline{\varpi}(\wp), \bar{\kappa}(\wp)+\bar{\varpi}(\wp)] \\
& (b)[c \odot \kappa]^{\wp}=c[\kappa]^{\wp}=\left\{\begin{array}{rll}
{[c \underline{\kappa}(\wp), c \bar{\kappa}(\wp)]} & \text { if } & c>0 \\
\{0\} & \text { if } & c=0 \\
{[c \bar{\kappa}(\wp), c \underline{\kappa}(\wp)]} & \text { if } & c<0
\end{array}\right. \\
& (c)[\kappa \odot \varpi]^{\wp}=\left[\begin{array}{l}
\min \{\underline{\kappa}(\wp) \underline{\varpi}(\wp), \underline{\kappa}(\wp) \bar{\varpi}(\wp), \bar{\kappa}(\wp) \underline{\varpi}(\wp), \bar{\kappa}(\wp) \bar{\varpi}(\wp)\}, \\
\max \{\underline{\kappa}(\wp) \underline{\varpi}(\wp), \underline{\kappa}(\wp) \bar{\varpi}(\wp), \bar{\kappa}(\wp) \underline{\varpi}(\wp), \bar{\kappa}(\wp) \bar{\varpi}(\wp)\}
\end{array}\right]
\end{aligned}
$$

where $\odot$ defines fuzzy multiplication.

The distance between fuzzy numbers $\kappa$ and $\varpi$ is given by the Hausdorff metric $D$ as

$$
D_{F}(\kappa, \varpi)=\underbrace{\sup }_{\wp \in[0,1]} D_{F}\left([\kappa]^{\wp},[\varpi]^{\wp}\right)=\underbrace{\sup }_{\wp \in[0,1]} \max \{|\underline{\kappa}(\wp)-\underline{\varpi}(\wp)|,|\bar{\kappa}(\wp)-\bar{\varpi}(\wp)|\}
$$

Thus $\left(\Lambda_{F}, D_{F}\right)$ defines a complete metric space with the properties of Hausdorff metric for fuzzy numbers as elaborated in [27].

The difference between any arbitrary fuzzy numbers is determined by the generalized Hukuhara difference or gH-difference for short as follows

\subsection{Generalized Hukuhara Difference}

Let $\kappa, \varpi \in \Lambda_{F}$ then gH-difference between $\kappa$ and $\varpi$ is defined as

$$
\kappa \ominus_{g H} \varpi=\sigma \Leftrightarrow\left\{\begin{array}{l}
(a) \kappa=\varpi+\sigma, \quad \text { or } \\
(b) \varpi=\kappa+(-1) \sigma
\end{array}\right.
$$

or in terms of $\wp$-level, for $\wp \in[0,1]$

$$
\kappa \ominus_{g H} \varpi=\left[\begin{array}{l}
\min \{\underline{\kappa}(\wp)-\underline{\varpi}(\wp), \bar{\kappa}(\wp)-\bar{\varpi}(\wp)\}, \\
\max \{\underline{\kappa}(\wp)-\underline{\varpi}(\wp), \bar{\kappa}(\wp)-\bar{\varpi}(\wp)\}
\end{array}\right]
$$

Furthermore, let $\ell\left([\kappa]^{\wp}\right)$ and $\ell\left([\varpi]^{\wp}\right)$ be length of $\kappa$ and $\varpi$ respectively, then 


$$
\begin{aligned}
& \text { (a) } \kappa \ominus_{g H} \varpi=\left[\begin{array}{c}
\underline{\kappa}(\wp)-\underline{\varpi}(\wp), \\
\bar{\kappa}(\wp)-\bar{\varpi}(\wp)
\end{array}\right] \quad \text { if } \quad \ell\left([\kappa]^{\wp}\right) \geq \ell\left([\varpi]^{\wp}\right) \\
& \text { (b) } \kappa \ominus_{g H} \varpi=\left[\begin{array}{c}
\bar{\kappa}(\wp)-\bar{\varpi}(\wp), \\
\underline{\kappa}(\wp)-\underline{\varpi}(\wp)
\end{array}\right] \quad \text { if } \quad \ell\left([\kappa]^{\wp}\right)<\ell\left([\varpi]^{\wp}\right)
\end{aligned}
$$

\subsection{Univariate and Bivariate Fuzzy-valued Function}

A fuzzy-valued function $\varphi$ is a rule that assigns to each $x \in \Re$ a unique fuzzy number $\varphi(x) \in \Lambda_{F}$. Its $\wp$-level representation is given by $\varphi(x ; \wp)=[\underline{\varphi}(x ; \wp), \bar{\varphi}(x ; \wp)], \forall x \in \Re$ and $\wp \in[0,1]$.

Fuzzy-valued function $\varphi$ of two variables $x, t \in \Re$ is defined as $\varphi:(x, t) \rightarrow \Lambda_{F}$, with its $\wp$-level representation $\varphi(x, t ; \wp)=[\underline{\varphi}(x, t ; \wp), \bar{\varphi}(x, t ; \wp)], \forall x \in \Re$ for all ordered pair $(x, t)$ of a set $O_{P}$ and for $\wp \in[0,1]$.

\subsection{Generalized Hukuhara Differentiability}

The gH-differentiability of fuzzy-valued functions was introduced by Bede et al. [28]. It is thoroughly defined for fuzzy-valued functions of two variables in Allahviranloo et al. [9]. Following the gH-difference, the gH-differentiability of univariate and bivariate fuzzy-valued function is defined as below.

A fuzzy-valued function $\varphi:(a, b) \rightarrow \Lambda_{F}$ is said to be gH-differentiable at $x_{0} \in(a, b)$ if $\varphi_{g H}^{\prime}\left(x_{0}\right) \in \Lambda_{F}$ exists such that

$$
\varphi_{g H}^{\prime}\left(x_{0}\right)=\lim _{h \rightarrow 0} \frac{\varphi\left(x_{0}+h\right) \ominus_{g H} \varphi\left(x_{0}\right)}{h}
$$

For $\varphi(x ; \wp)=[\underline{\varphi}(x ; \wp), \bar{\varphi}(x ; \wp)], \varphi$ is said to be gH-differentiable at $x$ if $\underline{\varphi}(x ; \wp)$ and $\bar{\varphi}(x ; \wp)$ are differentiable i.e.

$$
\varphi_{g H}^{\prime}(x)=\left[\min \left\{\frac{d}{d x} \underline{\varphi}(x ; \wp), \frac{d}{d x} \bar{\varphi}(x ; \wp)\right\}, \max \left\{\frac{d}{d x} \underline{\varphi}(x ; \wp), \frac{d}{d x} \bar{\varphi}(x ; \wp)\right\}\right]
$$

$\varphi$ is said to be $g H^{(i)}$-differentiable at $x$ i.e. $\varphi_{g H}^{\prime}(x ; \wp)=\left[\frac{d}{d x} \underline{\varphi}(x ; \wp), \frac{d}{d x} \bar{\varphi}(x ; \wp)\right]$ if $\ell(\varphi(x ; \wp))$ is increasing in $(a, b)$ for $\wp \in[0,1]$ and $g H^{(i i)}$-differentiable at $x$ i.e. $\varphi_{g H}^{\prime}(x ; \wp)=\left[\frac{d}{d x} \bar{\varphi}(x ; \wp), \frac{d}{d x} \underline{\varphi}(x ; \wp)\right]$ if $\ell(\varphi(x ; \wp))$ is decreasing in $(a, b)$ for $\wp \in[0,1]$.

A fuzzy-valued function $\varphi(x, t)$ is said to be gH-partially-differentiable $\left(g H_{p}\right.$-differentiable) at $\left(x_{0}, t_{0}\right) \in O_{P}$ with respect to the variables $x$ and $t$, if ${ }^{g H} \partial_{x} \varphi\left(x_{0}, t_{0}\right) \in \Lambda_{F}$ and ${ }^{g H} \partial_{t} \varphi\left(x_{0}, t_{0}\right) \in \Lambda_{F}$ exists, accordingly, i.e.

$$
\begin{aligned}
& { }^{g H} \partial_{x} \varphi\left(x_{0}, t_{0}\right)=\lim _{h \rightarrow 0} \frac{\varphi\left(x_{0}+h, t_{0}\right) \ominus_{g H} \varphi\left(x_{0}, t_{0}\right)}{h} \\
& { }^{g H} \partial_{t} \varphi\left(x_{0}, t_{0}\right)=\lim _{k \rightarrow 0} \frac{\varphi\left(x_{0}, t_{0}+k\right) \ominus_{g H} \varphi\left(x_{0}, t_{0}\right)}{k}
\end{aligned}
$$


Eqs. (5) and (6) can easily be computed similar to Eq. (3) by keeping $t$ fix for the fuzzy partial derivative with respect to $x$ and vice versa.

Similarly, $\varphi(x, t)$ is said to be $g H_{p}^{(i)}$-differentiable with respect to $x$ if $\ell(\varphi(x, t ; \wp))$ is increasing with respect to $x$ in $(a, b)$, for $\wp \in[0,1]$ i.e. ${ }^{g H} \partial_{x} \varphi(x, t ; \wp)=\left[\frac{\partial}{\partial x} \underline{\varphi}(x, t ; \wp), \frac{\partial}{\partial x} \bar{\varphi}(x, t ; \wp)\right]$ and $g H_{p}^{(i i)}$-differentiable if $\ell(\varphi(x, t ; \wp))$ is decreasing with respect to $x$ in $(a, b)$, for $\wp \in[0,1]$ i.e. ${ }^{g H} \partial_{x} \varphi(x, t ; \wp)=\left[\frac{\partial}{\partial x} \bar{\varphi}(x, t ; \wp), \frac{\partial}{\partial x} \underline{\varphi}(x, t ; \wp)\right]$ (same results for $g H_{p^{-}}$derivative of $\varphi(x, t)$ with respect to $t)$.

Let $\boldsymbol{C}_{F}$ be the space of all continuous fuzzy-valued functions, $\boldsymbol{A} \boldsymbol{C}_{F}$ be the space of all absolutely continuous fuzzy-valued functions and $\boldsymbol{L}_{F}$ the space of all Lebesgue integrable fuzzy-valued functions on $[a, b]$ then FRLFI and FCgHFD is defined as follows.

\subsection{Fuzzy Riemann-Liouville Fractional Integral}

Let $\varphi:[a, b] \rightarrow \Lambda_{F}$ then FRLFI of order $v>0$ is stated as

$$
\mathscr{I}_{a^{+}}^{v} \varphi(x)=\frac{1}{\Gamma v} \int_{a}^{x}(x-s)^{v-1} \varphi(s) d s \quad x>0
$$

and in form of $\wp$-level it is written as

$$
\mathscr{I}_{a^{+}}^{v} \varphi(x ; \wp)=\left[I_{a^{+}}^{v} \underline{\varphi}(x ; \wp), I_{a^{+}}^{v} \bar{\varphi}(x ; \wp)\right]
$$

where $I_{a^{+}}^{v} \underline{\varphi}(x ; \wp)=\frac{1}{\Gamma v} \int_{a}^{x}(x-s)^{v-1} \underline{\varphi}(s ; \wp) d s$

and $I_{a^{+}}^{v} \bar{\varphi}(x ; \wp)=\frac{1}{\Gamma v} \int_{a}^{x}(x-s)^{v-1} \bar{\varphi}(s ; \wp) d s$, for $\wp \in[0,1]$.

\subsection{Fuzzy Caputo gH-Fractional Derivative}

Let $\varphi(x) \in \boldsymbol{C}_{F} \cap \boldsymbol{L}_{F}$, then FCgHFD of order $\nu$ of fuzzy-valued function $\varphi(x)$ is defined as, for $x>0$ and $\lceil\nu\rceil-1<\nu \leq\lceil\nu\rceil$,

$$
{ }_{g H}^{C} \mathcal{D}_{a^{+}}^{\nu} \varphi(x)=\frac{1}{\Gamma(\lceil\nu\rceil-\nu)} \int_{a^{+}}^{x} \frac{\varphi_{g H}^{(\lceil\nu\rceil)}(s)}{(x-s)^{1-\lceil\nu\rceil+\nu}} d s
$$

where $\varphi_{g H}^{(m)}(s)$ is calculated as described in [28] and $\lceil\nu\rceil$ denote the smallest integer greater than or equal to $\nu$. If $\varphi(x ; \wp)=[\underline{\varphi}(x ; \wp), \bar{\varphi}(x ; \wp)]$ then for $\wp \in[0,1]$

$$
{ }_{g H}^{C} \mathcal{D}_{a^{+}}^{\nu} \varphi(x ; \wp)=\left[\begin{array}{c}
\min \left\{{ }_{g H}^{C} \mathrm{D}_{a^{+}}^{\nu} \underline{\varphi}(x ; \wp),{ }_{g H}^{C} \mathrm{D}_{a^{+}}^{\nu} \bar{\varphi}(x ; \wp)\right\} \\
\max \left\{{ }_{g H}^{C} \mathrm{D}_{a^{+}}^{\nu} \underline{\varphi}(x ; \wp),{ }_{g H}^{C} \mathrm{D}_{a^{+}}^{\nu} \bar{\varphi}(x ; \wp)\right\}
\end{array}\right]
$$

where

$$
{ }_{g H}^{C} \mathrm{D}_{a^{+}}^{\nu} \underline{\varphi}(x ; \wp)=\frac{1}{\Gamma(\lceil\nu\rceil-\nu)} \int_{a^{+}}^{x}(x-s)^{\lceil\nu\rceil-\nu-1} \frac{d^{\lceil\nu\rceil}}{d s^{\lceil\nu\rceil}} \underline{\varphi}(x ; \wp) d s
$$


and

$$
{ }_{g H}^{C} \mathrm{D}_{a^{+}}^{\nu} \bar{\varphi}(x ; \wp)=\frac{1}{\Gamma(\lceil\nu\rceil-\nu)} \int_{a^{+}}^{x}(x-s)^{\lceil\nu\rceil-\nu-1} \frac{d^{\lceil\nu\rceil}}{d s^{\lceil\nu\rceil}} \bar{\varphi}(x ; \wp) d s
$$

Now $\varphi:[a, b] \rightarrow \Lambda_{F}$ is said to be $F C g H F^{(i)}$-differentiable at $x \in[a, b]$ i.e. ${ }_{g H}^{C} \mathcal{D}_{a^{+}}^{\nu} \varphi(x ; \wp)=$ $\left.{ }_{g H}^{C} \mathrm{D}_{a^{+}}^{\nu} \underline{\varphi}(x ; \wp),{ }_{g H}^{C} \mathrm{D}_{a^{+}}^{\nu} \bar{\varphi}(x ; \wp)\right]$ if $\ell(\varphi(x ; \wp))$ is increasing in $[a, b]$ for $\wp \in[0,1]$ and $F C g H F^{(i i)}{ }_{-}$ differentiable i.e. ${ }_{g H}^{C} \mathcal{D}_{a^{+}}^{\nu} \varphi(x ; \wp)=\left[{ }_{g H}^{C} \mathrm{D}_{a^{+}}^{\nu} \bar{\varphi}(x ; \wp),{ }_{g H}^{C} \mathrm{D}_{a^{+}}^{\nu} \underline{\varphi}(x ; \wp)\right]$ if $\ell(\varphi(x ; \wp))$ is decreasing in $[a, b]$ for $\wp \in[0,1]$.

Consider $\varphi:(x, t) \rightarrow \Lambda_{F}$ with $x, t \in \Re$ then fuzzy Caputo gH-fractional partial derivative of order $\nu$ with respect to $x$ and $t$ of $\varphi(x, t)$ is determined as

$$
\begin{aligned}
& { }_{g H}^{C} \mathscr{D}_{x}^{\nu} \varphi(x, t)=\frac{1}{\Gamma(\lceil\nu\rceil-\nu)} \int_{a^{+}}^{x} \frac{{ }^{{ }^{+}} \partial_{x}^{(\lceil\nu\rceil)} \varphi_{g H}(s, t)}{(x-s)^{1-\lceil\nu\rceil+\nu}} d s, \quad x>0, \quad\lceil\nu\rceil-1<\nu \leq\lceil\nu\rceil \\
& { }_{g H}^{C} \mathscr{D}_{t}^{\nu} \varphi(x, t)=\frac{1}{\Gamma(\lceil\nu\rceil-\nu)} \int_{a^{+}}^{t} \frac{{ }^{{ }^{H}} \partial_{t}^{(\lceil\nu\rceil)} \varphi_{g H}(x, s)}{(t-s)^{1-\lceil\nu\rceil+\nu}} d s, \quad t>0, \quad\lceil\nu\rceil-1<\nu \leq\lceil\nu\rceil
\end{aligned}
$$

respectively. In case of $F C g H F_{P}^{(i)}$-differentiable and $F C g H F_{P}^{(i i)}$-differentiable of $\varphi(x, t)$, we consider $\ell(\varphi(x, t ; \wp))$ is increasing with respect to $x$ and $t$ both in $[a, b]$ and $\ell(\varphi(x, t ; \wp))$ is decreasing with respect to $x$ and $t$ both in $[a, b]$, accordingly, for $\wp \in[0,1]$.

The operator ${ }_{g H}^{C} \mathscr{D}_{t}^{\nu}$ satisfies the following properties that will be of an important use later. Since for any real valued function $\eta(x, t)$

$$
I_{t}^{\nu C} \mathrm{D}_{t}^{\nu} \eta(x, t)=\eta(x, t)-\sum_{l=0}^{\lceil\nu\rceil-1} \partial_{t}^{(l)} \eta(x, 0) \frac{t^{l}}{l !}
$$

then for $\varphi(x, t) \in \Lambda_{F}$ and $\wp \in[0,1]$.

$$
\mathscr{I}_{t}^{\nu C} \mathscr{D}_{t}^{\nu} \varphi(x, t ; \wp)=\varphi(x, t ; \wp) \ominus \sum_{l=0}^{\lceil\nu\rceil-1}{ }_{g H} \partial_{t}^{(l)} \varphi_{g H}(x, 0 ; \wp) \frac{t^{l}}{l !}
$$

If $\varphi(x, t)$ is $F C g H F_{P}^{(i)}$-differentiable and

$$
\mathscr{I}_{t}^{\nu C} \mathscr{D}_{t}^{\nu} \varphi(x, t ; \wp)=-\sum_{l=0}^{\lceil\nu\rceil-1}{ }_{g H} \partial_{t}^{(l)} \varphi_{g H}(x, 0 ; \wp) \frac{t^{l}}{l !} \ominus(-\varphi(x, t ; \wp))
$$

when $\varphi(x, t)$ is $F C g H F_{P}^{(i i)}$-differentiable. 


\section{Shifted Chebyshev Polynomials and Operational Matrices}

In this section, SCPs are defined for the approximation of fuzzy-valued functions along with the SCOM of Caputo fractional derivative and SCOM of Riemann-Liouville fractional integration. The detailed derivation of SCOMs is given in [22, 23].

\subsection{Shifted Chebyshev Polynomial}

The Chebyshev polynomials $\mathcal{T}_{i}$ for $i=0,1, \ldots$ are defined on $(-1,1)$. In order to make these polynomials functional on the interval $\xi \in(0, L)$, it is shifted by introducing the change of variable $\varsigma=\frac{2 \xi}{L}-1$. Let the polynomials $\mathcal{T}_{i}\left(\frac{2 \xi}{L}-1\right)$, named as shifted Chebyshev polynomials be represented by $\mathcal{T}_{L, i}(\xi)$, with the orthogonal property

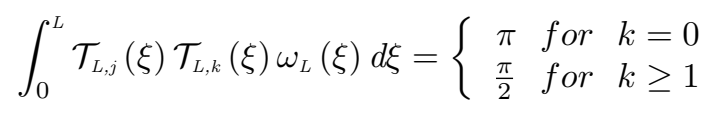

where $\omega_{L}(\xi)=\frac{1}{\sqrt{L \xi-\xi^{2}}}$

$$
\mathcal{T}_{L, i}(\xi)=i \sum_{k=0}^{i}(-1)^{i-k} \frac{(i+k-1) ! 2^{2 k}}{(i-k) !(2 k) ! L^{k}} \xi^{k}
$$

where $\mathcal{T}_{L, i}(0)=(-1)^{i}$ and $\mathcal{T}_{L, i}(L)=1$.

In this form, $\mathcal{T}_{L, i}(\xi)$ may be generated with the aid of the following recurrence formula

$$
\mathcal{T}_{L, i+1}(\xi)=2\left(\frac{2 \xi}{L}-1\right) \mathcal{T}_{L, i}(\xi)-\mathcal{T}_{L, i-1}(\xi) \quad i=1,2, \ldots
$$

where $\mathcal{T}_{L, 0}(\xi)=1$ and $\mathcal{T}_{L, 1}(\xi)=\frac{2 \xi}{L}-1$

Any function y $(\xi)$, square integrable in $(0, L)$, can be expressed in terms of the SCPs as

$$
\mathrm{y}(\xi)=\sum_{j=0}^{\infty} \alpha_{j} \mathcal{T}_{L, j}(\xi)
$$

where

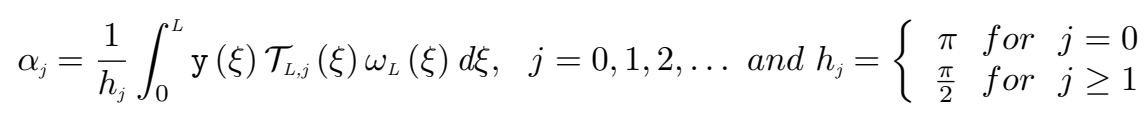

In practice, only the first $(N+1)$ terms of SCPs are considered thus y $(\xi)$ can be approximated as the truncated series 


$$
\mathrm{y}_{N}(\xi) \cong \sum_{j=0}^{N} \alpha_{j} \mathcal{T}_{L, j}(\xi)=\mathbf{A}^{T} \Psi_{N}(\xi)
$$

where the shifted Chebyshev coefficient vector $\mathbf{A}$ and the vector $\Psi_{N}(\xi)$ are given by

$$
\mathbf{A}^{T}=\left[\alpha_{0}, \alpha_{1}, \ldots, \alpha_{N}\right], \quad \Psi_{N}(\xi)=\left[\mathcal{T}_{L, 0}(\xi), \mathcal{T}_{L, 1}(\xi), \ldots, \mathcal{T}_{L, N}(\xi)\right]
$$

Similarly, Let $\mathrm{y}(\xi, t) \in[0, L) \times[0, L)$ then it maybe expressed as

$$
\mathrm{y}_{M, N}(\xi, t)=\sum_{m=0}^{M} \sum_{n=0}^{N} \beta_{m n} \mathcal{T}_{L, m}(t) \mathcal{T}_{L, n}(\xi)=\Psi_{M}^{T}(t) \mathbf{B} \Psi_{N}(\xi)
$$

with

$$
\begin{gathered}
\mathbf{B}=\left[\begin{array}{rrrr}
\beta_{00} & \beta_{01} & \cdots & \beta_{0 N} \\
\beta_{10} & \beta_{11} & \cdots & \beta_{1 N} \\
\vdots & \vdots & \cdots & \vdots \\
\beta_{M 0} & \beta_{M 1} & \cdots & \beta_{M N}
\end{array}\right] \\
\beta_{m n}=\left(\frac{1}{h_{m}}\right)\left(\frac{1}{h_{n}}\right) \iint_{0}^{L} \mathrm{y}_{M, N}(\xi, t) \mathcal{T}_{L, m}(t) \omega_{L}(t) \mathcal{T}_{L, n}(\xi) \omega_{L}(\xi) d \xi d t
\end{gathered}
$$

where $m=0,1, \ldots, M$ and $n=0,1, \ldots, N$.

\subsection{Shifted Chebyshev Operational Matrix of Fractional Integration and Derivative}

Theorem 3.2.1 Let $\Psi_{M}(t)$ be the shifted Chebyshev vector then for $v>0$

$$
I^{v} \Psi_{M}(t) \cong \mathbf{P}^{(v)} \Psi_{M}(t)
$$

where $\mathbf{P}^{(v)}$ is the $(M+1) \times(M+1)$ operational matrix of fractional integration of order $v$ in the Riemann-Liouville sense and is defined as follows

$$
\mathbf{P}^{(v)}=\left[\begin{array}{rrrrr}
H_{v}(0,0) & H_{v}(0,1) & H_{v}(0,2) & \cdots & H_{v}(0, M) \\
H_{v}(1,0) & H_{v}(1,1) & H_{v}(1,2) & \cdots & H_{v}(1, M) \\
\vdots & \vdots & \vdots & \cdots & \vdots \\
H_{v}(m, 0) & H_{v}(m, 1) & H_{v}(m, 2) & \cdots & H_{v}(m, M) \\
\vdots & \vdots & \vdots & \cdots & \vdots \\
H_{v}(M, 0) & H_{v}(M, 1) & H_{v}(M, 2) & \cdots & H_{v}(M, M)
\end{array}\right]
$$


where

$$
H_{v}(m, j)=\sum_{k=0}^{m} \frac{(-1)^{i-k} L^{v}(m+k-1) ! \Gamma\left(k+v+\frac{1}{2}\right)}{e_{j} \Gamma\left(k+\frac{1}{2}\right)(m-k) ! \Gamma(k+v-j+1) \Gamma(k+j+v+1)}
$$

with $e_{j}=\left\{\begin{array}{ll}2 & \text { for } j=0 \\ 1 & \text { for } j \geq 1\end{array}\right.$.

Theorem 3.2.2 Let $\Psi_{N}(\xi)$ be the shifted Chebyshev vector, then Caputo fractional derivative of order $\nu$ of $\Psi_{N}(\xi)$ is given by

$$
{ }^{C} \mathrm{D}^{\nu} \Psi_{N}(\xi) \cong \mathbf{D}^{(\nu)} \Psi_{N}(\xi), \quad\lceil\nu\rceil-1<\nu \leq\lceil\nu\rceil
$$

where $\mathbf{D}^{(\nu)}$ is the $(N+1) \times(N+1)$ operational matrix of Caputo fractional derivative and is given by

$$
\mathbf{D}^{(\nu)}=\left[\begin{array}{rrrlr}
0 & 0 & 0 & \cdots & 0 \\
\vdots & \vdots & \vdots & \cdots & \vdots \\
0 & 0 & 0 & \cdots & 0 \\
G_{\nu}(\lceil\nu\rceil, 0) & G_{\nu}(\lceil\nu\rceil, 1) & G_{\nu}(\lceil\nu\rceil, 2) & \cdots & G_{\nu}(\lceil\nu\rceil, N) \\
\vdots & \vdots & \vdots & \cdots & \vdots \\
G_{\nu}(n, 0) & G_{\nu}(n, 1) & G_{\nu}(n, 2) & \cdots & G_{\nu}(n, N) \\
\vdots & \vdots & \vdots & \cdots & \vdots \\
G_{\nu}(N, 0) & G_{\nu}(N, 1) & G_{\nu}(N, 2) & \cdots & G_{\nu}(N, N)
\end{array}\right]
$$

where

$$
G_{\nu}(n, j)=\sum_{k=\lceil\nu\rceil}^{n} \frac{(-1)^{i-k} L^{-\nu} 2 i(n+k-1) ! \Gamma\left(k-\nu+\frac{1}{2}\right)}{e_{j} \Gamma\left(k+\frac{1}{2}\right)(m-k) ! \Gamma(k-\nu-j+1) \Gamma(k+j-\nu+1)}
$$

with $e_{j}=\left\{\begin{array}{l}2 \text { for } j=0 \\ 1 \text { for } j \geq 1\end{array}\right.$.

Lemma 1. Let $\mathcal{T}_{L, j}(\xi)$ be a SCPs then.

$$
{ }^{C} \mathrm{D}^{\nu} \mathcal{T}_{L, j}(\xi)=0, \quad j=0,1, \ldots,\lceil\nu\rceil-1, \quad \nu>0
$$




\section{Fuzzy-valued Function Approximation}

Consider fuzzy-valued function $\varphi(\xi) \in \boldsymbol{C}_{F}[0, L] \bigcap \boldsymbol{L}_{F}[0, L]$, then $\varphi(\xi)$ can be approximated by the linear combination of SCP as

$$
\varphi(\xi)=\sum_{j=0}^{\infty}{ }^{*} \sigma_{j} \odot \mathcal{T}_{L, j}(\xi), \quad \xi \in[0, L]
$$

where fuzzy coefficients $\sigma_{j}$ are obtained by

$$
\sigma_{j}=\frac{1}{h_{j}} \int_{0}^{L} \varphi(\xi) \mathcal{T}_{L, j}(\xi) \omega_{L}(\xi) d \xi, \quad j=0,1,2 \ldots
$$

In Eq. (33) $\sum^{*}$ and $\odot$ means addition with respect to $\oplus$ and multiplication in $\Lambda_{F}$, respectively. Eq. (33) can be written in truncated form as

$$
\varphi(\xi) \cong \varphi_{N}(\xi)=\sum_{j=0}^{\infty}{ }^{*} \sigma_{j} \odot \mathcal{T}_{L, j}(\xi)=\mathbf{H}^{T} \odot \Psi_{N}(\xi)
$$

which can also be written in $\wp$-level as, for $\wp \in[0,1]$

$$
\begin{array}{r}
\varphi_{N}(\xi ; \wp)=\left[\underline{\varphi}_{N}(\xi ; \wp), \bar{\varphi}_{N}(\xi ; \wp)\right]=\left[\sum_{j=0}^{\infty} \underline{\sigma}_{j}(\wp) \mathcal{T}_{L, j}(\xi), \sum_{j=0}^{\infty} \bar{\sigma}_{j}(\wp) \mathcal{T}_{L, j}(\xi)\right] \\
=\left[\underline{\mathbf{H}}(\wp)^{T} \Psi_{N}(\xi), \overline{\mathbf{H}}(\wp)^{T} \Psi_{N}(\xi)\right]
\end{array}
$$

So that $\Psi_{N}(\xi)$ is obtained as given in Eq. (22) and fuzzy shifted Chebyshev coefficient vectors $\underline{\mathbf{H}}(\wp)^{T}$ and $\overline{\mathbf{H}}(\wp)^{T}$ are determined as

$$
\underline{\mathbf{H}}(\wp)^{T}=\left[\underline{\sigma}_{0}(\wp), \underline{\sigma}_{1}(\wp), \ldots, \underline{\sigma}_{N}(\wp)\right], \quad \overline{\mathbf{H}}(\wp)^{T}=\left[\bar{\sigma}_{0}(\wp), \bar{\sigma}_{1}(\wp), \ldots, \bar{\sigma}_{N}(\wp)\right]
$$

Now consider fuzzy-valued function $\varphi(\xi, t ; \wp) \in \boldsymbol{C}_{F}[0, L] \bigcap \boldsymbol{L}_{F}[0, L]$, then it can be written as linear combination of SCPs same as in Eq. (23) i.e.

$$
\begin{gathered}
\varphi_{M, N}(\xi, t)=\sum_{m=0}^{M} \sum_{n=0}^{N}{ }^{*} \varepsilon_{m n} \odot \mathcal{T}_{L, m}(t) \mathcal{T}_{L, n}(\xi)=\Psi_{M}^{T}(t) \mathbf{K}(\wp) \Psi_{N}(\xi) \\
m=0,1, \ldots, M, n=0,1, \ldots, N
\end{gathered}
$$

where $\Psi_{M}^{T}(t), \Psi_{N}(\xi)$ are same as in Eq. $(22)$.

Let $\varphi(\xi, t ; \wp)=[\varphi(\xi, t ; \wp), \bar{\varphi}(\xi, t ; \wp)]$ for $\wp \in[0,1]$, then its lower and upper functions are approximated as 


$$
\begin{gathered}
\varphi_{M, N}(\xi, t ; \wp)=\left[\underline{\varphi}_{M, N}(\xi, t ; \wp), \bar{\varphi}_{M, N}(\xi, t ; \wp)\right]=\left[\begin{array}{c}
\sum_{m=0}^{M} \sum_{n=0}^{N} \underline{\varepsilon}_{m n} \mathcal{T}_{L, m}(t) \mathcal{T}_{L, n}(\xi), \\
\sum_{m=0}^{M} \sum_{n=0}^{N} \bar{\varepsilon}_{m n} \mathcal{T}_{L, m}(t) \mathcal{T}_{L, n}(\xi)
\end{array}\right] \\
=\left[\Psi_{M}^{T}(t) \underline{\mathbf{K}}(\wp) \Psi_{N}(\xi), \Psi_{M}^{T}(t) \overline{\mathbf{K}}(\wp) \Psi_{N}(\xi)\right]
\end{gathered}
$$

with fuzzy shifted Chebyshev coefficients

$$
\begin{array}{r}
\underline{\mathbf{K}}(\wp)=\left[\begin{array}{rrrr}
\underline{\varepsilon}_{00}(\wp) & \underline{\varepsilon}_{01}(\wp) & \cdots & \underline{\varepsilon}_{0 N}(\wp) \\
\underline{\varepsilon}_{10}(\wp) & \underline{\varepsilon}_{11}(\wp) & \cdots & \underline{\varepsilon}_{1 N}(\wp) \\
\vdots & \vdots & \cdots & \vdots \\
\underline{\varepsilon}_{M 0}(\wp) & \underline{\varepsilon}_{M 1}(\wp) & \cdots & \underline{\varepsilon}_{M N}(\wp)
\end{array}\right], \\
\overline{\mathbf{K}}(\wp)=\left[\begin{array}{rrrr}
\bar{\varepsilon}_{00}(\wp) & \bar{\varepsilon}_{01}(\wp) & \ldots & \bar{\varepsilon}_{0 N}(\wp) \\
\bar{\varepsilon}_{10}(\wp) & \bar{\varepsilon}_{11}(\wp) & \cdots & \bar{\varepsilon}_{1 N}(\wp) \\
\vdots & \vdots & \ldots & \vdots \\
\bar{\varepsilon}_{M 0}(\wp) & \bar{\varepsilon}_{M 1}(\wp) & \cdots & \bar{\varepsilon}_{M N}(\wp)
\end{array}\right]
\end{array}
$$

where $\bar{\varepsilon}_{m n}(\wp)$ and $\bar{\varepsilon}_{m n}(\wp)$ are calculated in the same way as defined in Eq. (25).

\section{Proposed Technique}

In this section, the shifted Chebyshev spectral-tau method is elaborated for the numerical solution of FFDE and FFWE of space and time fractional, separately. See ref.[23].

\subsection{Fuzzy Fractional Diffusion Equation}

Let $\varphi(\xi, t ; \wp) \in \boldsymbol{C}_{F}[0, L] \cap \boldsymbol{L}_{F}[0, L]$, consider the following time and space fractional fuzzy diffusion equation

$$
{ }_{g H}^{C} \mathscr{D}_{t}^{\nu} \varphi(\xi, t) \ominus_{g H}^{C} \mathscr{D}_{\xi}^{\lambda} \varphi(\xi, t)=\varphi(\xi, t) \quad \xi, t \in[0, L], \quad 0<\nu \leq 1, \quad 0<\lambda \leq 2
$$

with boundary conditions

$$
\varphi(\xi, 0)=\tilde{g}(\xi), \quad \varphi(0, t)=\tilde{h}^{1}(t), \quad \varphi(1, t)=\tilde{h}^{2}(t)
$$

Integrate Eq. (41) using Eqs. (14) and (15), we get the following equations for $\wp \in[0,1]$

$$
\varphi(\xi, t ; \wp) \ominus \varphi(\xi, 0 ; \wp) \ominus \mathscr{I}_{t}^{\nu C}{ }_{g H} \mathscr{D}_{\xi}^{\lambda} \varphi(\xi, t ; \wp)=\mathscr{I}_{t}^{\nu} \varphi(\xi, t ; \wp)
$$


or

$$
-\varphi(\xi, 0 ; \wp) \ominus(-\varphi(\xi, t ; \wp)) \oplus\left(-\mathscr{I}_{t}^{\nu C} \mathscr{D}_{\xi}^{\lambda} \varphi(\xi, t ; \wp)\right)=\ominus\left(-\mathscr{I}_{t}^{\nu} \varphi(\xi, t ; \wp)\right)
$$

Use SCPs and SCOM of fractional integral and Caputo fractional derivative to approximate Eq. (43) as, for $\wp \in[0,1]$,

$$
\begin{gathered}
\varphi_{M, N}(\xi, t ; \wp)=\Psi_{M}^{T}(t) \odot \mathbf{K}(\wp) \odot \Psi_{N}(\xi), \\
\tilde{\mathrm{g}}(\xi)=\mathbf{G}(\wp) \odot \Psi_{N}(\xi)
\end{gathered}
$$

and in $\wp$-level

$$
\begin{gathered}
\varphi_{M, N}(\xi, t ; \wp)=\left[\Psi_{M}^{T}(t) \underline{\mathbf{K}}(\wp) \Psi_{N}(\xi), \Psi_{M}^{T}(t) \overline{\mathbf{K}}(\wp) \Psi_{N}(\xi)\right], \\
\tilde{\mathrm{g}}(\xi ; \wp)=\left[\underline{\mathbf{G}}(\wp) \Psi_{N}(\xi), \overline{\mathbf{G}}(\wp) \Psi_{N}(\xi)\right]
\end{gathered}
$$

where $\underline{\mathbf{K}}(\wp)$ and $\overline{\mathbf{K}}(\wp)$ are defined as in Eq. (40)and $\mathbf{G}(\wp)$ is obtained as

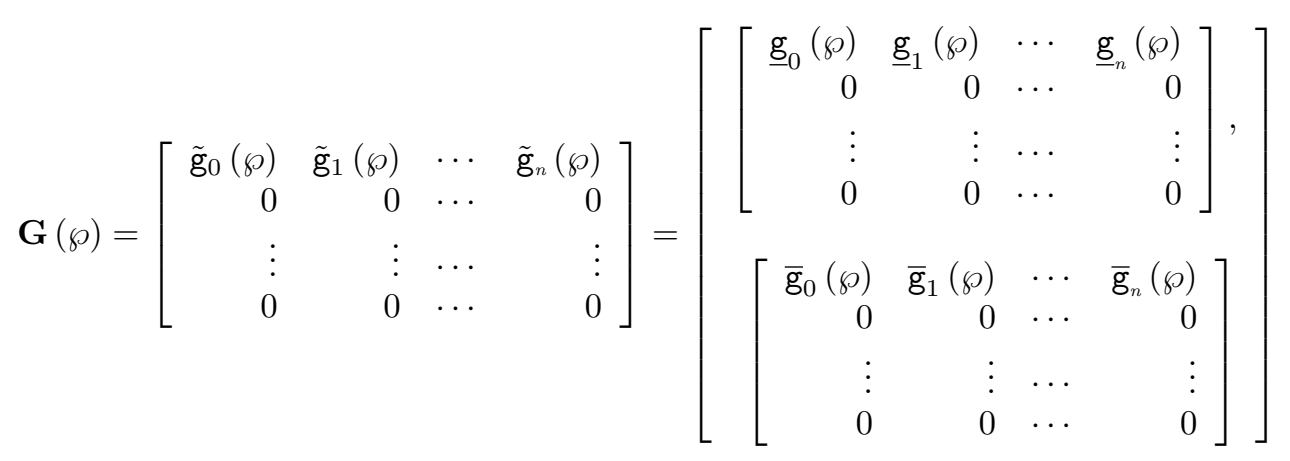

where $\underline{\mathrm{g}}_{n}(\wp)$ and $\overline{\mathrm{g}}_{n}(\wp)$ are same as in Eq. (20). In addition, using Eq. (27) and (30) we can approximate $\mathscr{I}_{t}^{\nu C}{ }_{g H} \mathscr{D}_{\xi}^{\lambda} \varphi(\xi, t ; \wp)$ and $\mathscr{I}_{t}^{\nu} \varphi(\xi, t ; \wp)$ as

$$
\begin{gathered}
\mathscr{I}_{t g h}^{\nu C} \mathscr{D}_{\xi}^{\lambda} \varphi(\xi, t ; \wp)=\Psi_{M}^{T}(t) \mathbf{P}^{(v)^{T}} \odot \mathbf{K}(\wp) \odot \mathbf{D}^{(\lambda)} \Psi_{N}(\xi) \\
\mathscr{I}_{t}^{\nu} \varphi(\xi, t ; \wp)=\Psi_{M}^{T}(t) \mathbf{P}^{(v)^{T}} \odot \mathbf{K}(\wp) \odot \Psi_{N}(\xi)
\end{gathered}
$$

Substitute Eqs. (45), (46), (50) and (51) in Eq. (43) we get the residual $\tilde{\mathbf{R}}_{M, N}(\xi, t)$ for Eq. (43) in the form 


$$
\tilde{\mathbf{R}}_{M, N}(\xi, t)=\Psi_{M}^{T}(t)\left(\mathbf{K}(\wp) \ominus \mathbf{G}(\wp) \ominus \mathbf{P}^{(v)^{T}} \odot \mathbf{K}(\wp) \odot \mathbf{D}^{(\lambda)} \ominus \mathbf{P}^{(v)^{T}} \odot \mathbf{K}(\wp)\right) \Psi_{N}(\xi)
$$

which can be written in $\wp$-level form as

$$
\underline{\mathbf{R}}_{M, N}(\xi, t ; \wp)=\Psi_{M}^{T}(t)\left(\underline{\mathbf{K}}(\wp)-\underline{\mathbf{G}}(\wp)-\mathbf{P}^{(v)^{T}} \underline{\mathbf{K}}(\wp) \mathbf{D}^{(\lambda)}-\mathbf{P}^{(v)^{T}} \underline{\mathbf{K}}(\wp)\right) \Psi_{N}(\xi)
$$

and

$$
\overline{\mathbf{R}}_{M, N}(\xi, t ; \wp)=\Psi_{M}^{T}(t)\left(\overline{\mathbf{K}}(\wp)-\overline{\mathbf{G}}(\wp)-\mathbf{P}^{(v)^{T}} \overline{\mathbf{K}}(\wp) \mathbf{D}^{(\lambda)}-\mathbf{P}^{(v)^{T}} \overline{\mathbf{K}}(\wp)\right) \Psi_{N}(\xi)
$$

where $\underline{\mathbf{R}}_{M, N}(\xi, t ; \wp)$ and $\overline{\mathbf{R}}_{M, N}(\xi, t ; \wp)$ represent the residual for lower and upper functions, respectively, $\underline{\mathbf{K}}(\wp)$ and $\overline{\mathbf{K}}(\wp)$ are described in Eq. (40). As in a typical tau method, $(M+1)(N-1)$ fuzzy linear algebraic equations in the unknown expansion coefficients $\varepsilon_{m n}(\wp), m=0,1, \ldots, M$, $n=0,1, \ldots, N$ are generated by

$$
\iint_{0}^{L} \underline{\mathbf{R}}_{M, N}(\xi, t ; \wp) \mathcal{T}_{L, m}(t) \mathcal{T}_{L, n}(\xi) d \xi d t=0
$$

for lower function and

$$
\iint_{0}^{L} \overline{\mathbf{R}}_{M, N}(\xi, t ; \wp) \mathcal{T}_{L, m}(t) \mathcal{T}_{L, n}(\xi) d \xi d t=0
$$

for upper function, for $m=0,1, \ldots, M, n=0,1, \ldots, N-2$.

The rest of the fuzzy linear algebraic equations are obtained from the boundary conditions as

$$
\Psi_{M}^{T}(t) \underline{\mathbf{K}}(\wp) \Psi_{N}(\xi)=\underline{h}^{1}\left(t_{m} ; \wp\right), \quad \Psi_{M}^{T}(t) \overline{\mathbf{K}}(\wp) \Psi_{N}(\xi)=\bar{h}^{1}\left(t_{m} ; \wp\right)
$$

and

$$
\Psi_{M}^{T}(t) \underline{\mathbf{K}}(\wp) \Psi_{N}(\xi)=\underline{h}^{2}\left(t_{m} ; \wp\right), \quad \Psi_{M}^{T}(t) \overline{\mathbf{K}}(\wp) \Psi_{N}(\xi)=\bar{h}^{2}\left(t_{m} ; \wp\right)
$$

Where $t_{m}, m=0,1, \ldots, M$ are the roots of $\mathcal{T}_{L, M+1}(t)$. Thus from fuzzy linear equations (55)-(58), $(M+1)(N+1)$ unknown fuzzy coefficients $\underline{\varepsilon}_{m n}(\wp)$ and $\bar{\varepsilon}_{m n}(\wp)$ are calculated and so approximate solution $\varphi_{M, N}(\xi, t ; \wp)$ for $F C g H F_{P}^{(i)}$-differentiability is obtained from Eq. (47).

For Eq. (44), same approximations are taken into account as defined in Eq. (45)-(51) and following same algorithm and using the residuals 


$$
\underline{\mathbf{R}}_{M, N}(\xi, t ; \wp)=\Psi_{M}^{T}(t)\left(-\underline{\mathbf{G}}(\wp)-\overline{\mathbf{K}}(\wp)-\mathbf{P}^{(v)^{T}} \overline{\mathbf{K}}(\wp) \mathbf{D}^{(\lambda)}-\mathbf{P}^{(v)^{T}} \overline{\mathbf{K}}(\wp)\right) \Psi_{N}(\xi)
$$

for lower function and

$$
\overline{\mathbf{R}}_{M, N}(\xi, t ; \wp)=\Psi_{M}^{T}(t)\left(-\overline{\mathbf{G}}(\wp)-\underline{\mathbf{K}}(\wp)-\mathbf{P}^{(v)^{T}} \underline{\mathbf{K}}(\wp) \mathbf{D}^{(\lambda)}-\mathbf{P}^{(v)^{T}} \underline{\mathbf{K}}(\wp)\right) \Psi_{N}(\xi)
$$

for upper function and approximate solution $\varphi_{M, N}(\xi, t ; \wp)$ for $F C g H F_{P}^{(i i)}$-differentiability is obtained.

\subsection{Fuzzy Fractional Wave Equation}

Now consider the following time and space fractional fuzzy wave equation

$$
{ }_{g H}^{C} \mathscr{D}_{t}^{\nu} \varphi(\xi, t) \oplus_{g H}^{C} \mathscr{D}_{\xi}^{\lambda} \varphi(\xi, t)=\tilde{f}(\xi, t) \quad \xi, t \in[0, L], 0<\nu \leq 2,0<\lambda \leq 2
$$

where $\tilde{f}(\xi, t) \in \Lambda_{F}$ with boundary conditions

$$
\varphi(\xi, 0)=\tilde{\mathrm{g}}(\xi),{ }^{g H} \partial_{t} \varphi(\xi, 0)=\tilde{h}^{1}(t), \quad \varphi(1, t)=\tilde{h}^{2}(t)
$$

On integrating Eq. (61) using Eqs. (14) and (15), we have the following equations for $\wp \in[0,1]$

$$
\varphi(\xi, t ; \wp) \ominus \varphi(\xi, 0 ; \wp) \ominus^{g H} \partial_{t} \varphi(\xi, 0) t \oplus \mathscr{I}_{t}^{\nu C} \mathscr{D}_{\xi}^{\lambda} \varphi(\xi, t ; \wp)=\mathscr{I}_{t}^{\nu} \tilde{f}(\xi, t ; \wp)
$$

or

$$
-\varphi(\xi, t ; \wp)-{ }^{g H} \partial_{t} \varphi(\xi, 0) t \ominus(-\varphi(\xi, 0 ; \wp)) \ominus\left(-\mathscr{I}_{t}^{\nu C}{ }_{g H}^{\nu} \mathscr{D}_{\xi}^{\lambda} \varphi(\xi, t ; \wp)\right)=\ominus\left(-\mathscr{I}_{t}^{\nu} \tilde{f}(\xi, t ; \wp)\right)
$$

Consider same approximations of $\varphi_{M, N}(\xi, t ; \wp), \tilde{\mathrm{g}}_{N}(\xi)$ and $\mathscr{I}_{t}^{\nu C} \mathscr{D}_{\xi}^{\nu} \varphi(\xi, t ; \wp)$ as described in Eqs. (45)-(51) in previous section with

$$
\tilde{f}(\xi, t ; \wp)=\Psi_{M}^{T}(t) \odot \mathbf{F}(\wp) \odot \Psi_{N}(\xi)
$$

in $\wp$-level, for $\wp \in[0,1]$,

$$
\tilde{f}(\xi, t ; \wp)=\left[\Psi_{M}^{T}(t) \underline{\mathbf{F}}(\wp) \Psi_{N}(\xi), \Psi_{M}^{T}(t) \overline{\mathbf{F}}(\wp) \Psi_{N}(\xi)\right]
$$

where $\mathbf{F}(\wp)$ is 


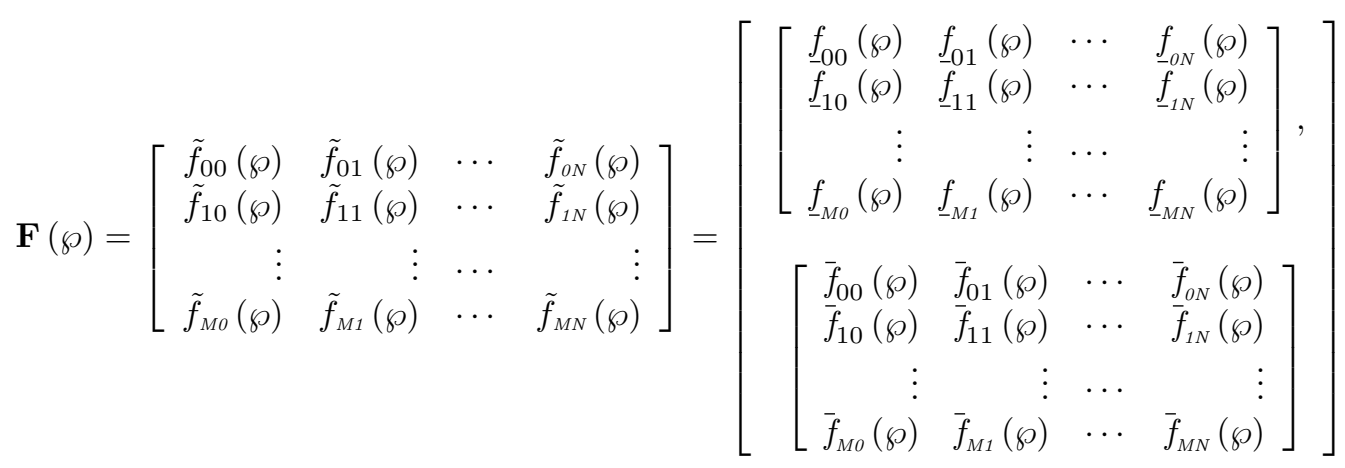

where $f_{-m n}(\wp)$ and $\bar{f}_{m n}(\wp)$ are same as in Eq. (25). Moreover, using Eq. $(27), \mathscr{I}_{t}^{\nu} \tilde{f}(\xi, t)$ is approximated as

$$
\mathscr{I}_{t}^{\nu} \tilde{f}(\xi, t)=\Psi_{M}^{T}(t) \mathbf{P}^{(v)^{T}} \odot \mathbf{F}(\wp) \odot \Psi_{N}(\xi)
$$

On substitution of Eqs. (45), (46), (50), (51) and (68) in Eq. (63) we get the residual $\tilde{\mathbf{R}}_{M, N}(\xi, t)$ for Eq. (63) in the form

$$
\tilde{\mathbf{R}}_{M, N}(\xi, t)=\Psi_{M}^{T}(t)\left(\mathbf{K}(\wp) \ominus \mathbf{G}(\wp) \oplus \mathbf{P}^{(v)^{T}} \odot \mathbf{K}(\wp) \odot \mathbf{D}^{(\lambda)} \ominus \mathbf{P}^{(v)^{T}} \odot \mathbf{F}(\wp)\right) \Psi_{N}(\xi)
$$

and in its $\wp$-level form as

$$
\underline{\mathbf{R}}_{M, N}(\xi, t ; \wp)=\Psi_{M}^{T}(t)\left(\underline{\mathbf{K}}(\wp)-\underline{\mathbf{G}}(\wp)+\mathbf{P}^{(v)^{T}} \underline{\mathbf{K}}(\wp) \mathbf{D}^{(\lambda)}-\mathbf{P}^{(v)^{T}} \underline{\mathbf{F}}(\wp)\right) \Psi_{N}(\xi)
$$

and

$$
\overline{\mathbf{R}}_{M, N}(\xi, t ; \wp)=\Psi_{M}^{T}(t)\left(\overline{\mathbf{K}}(\wp)-\overline{\mathbf{G}}(\wp)+\mathbf{P}^{(v)^{T}} \overline{\mathbf{K}}(\wp) \mathbf{D}^{(\lambda)}-\mathbf{P}^{(v)^{T}} \overline{\mathbf{F}}(\wp)\right) \Psi_{N}(\xi)
$$

Hence, $(M+1)(N-1)$ fuzzy linear algebraic equations in the unknown expansion coefficients $\varepsilon_{m n}(\wp), m=0,1, \ldots, M, n=0,1, \ldots, N$ are generated by

$$
\iint_{0}^{L} \underline{\mathbf{R}}_{M, N}(\xi, t ; \wp) \mathcal{T}_{L, m}(t) \mathcal{T}_{L, n}(\xi) d \xi d t=0
$$

for lower function and

$$
\iint_{0}^{L} \overline{\mathbf{R}}_{M, N}(\xi, t ; \wp) \mathcal{T}_{L, m}(t) \mathcal{T}_{L, n}(\xi) d \xi d t=0
$$


for upper function, for $m=0,1, \ldots, M, n=0,1, \ldots, N-2$ and remaining equations from the boundary conditions

$$
\Psi_{M}^{T}(t) \underline{\mathbf{K}}(\wp) \Psi_{N}(\xi)=\underline{h}^{1}\left(t_{m} ; \wp\right), \quad \Psi_{M}^{T}(t) \overline{\mathbf{K}}(\wp) \Psi_{N}(\xi)=\bar{h}^{1}\left(t_{m} ; \wp\right)
$$

and

$$
\Psi_{M}^{T}(t) \underline{\mathbf{K}}(\wp) \Psi_{N}(\xi)=\underline{h}^{2}\left(t_{m} ; \wp\right), \quad \Psi_{M}^{T}(t) \overline{\mathbf{K}}(\wp) \Psi_{N}(\xi)=\bar{h}^{2}\left(t_{m} ; \wp\right)
$$

where $t_{m}, m=0,1, \ldots, M$ are the roots of $\mathcal{T}_{L, M+1}(t)$. Thus, $(M+1)(N+1)$ unknown fuzzy coefficients $\underline{\varepsilon}_{m n}(\wp)$ and $\bar{\varepsilon}_{m n}(\wp)$ are calculated. Consequently, approximate solution $\varphi_{M, N}(\xi, t ; \wp)$ for $F C g H F_{P}^{(i)}$-differentiability is obtained from Eq. (47).

For Eq. (64), same approximations are taken into account as defined in Eqs. (45)-(51) and Eqs. (65)-(68). Following same algorithm and on using the residuals

$$
\underline{\mathbf{R}}_{M, N}(\xi, t ; \wp)=\Psi_{M}^{T}(t)\left(-\underline{\mathbf{G}}(\wp)-\overline{\mathbf{K}}(\wp)+\mathbf{P}^{(v)^{T}} \overline{\mathbf{K}}(\wp) \mathbf{D}^{(\lambda)}-\mathbf{P}^{(v)^{T}} \overline{\mathbf{F}}(\wp)\right) \Psi_{N}(\xi)
$$

for lower function and

$$
\overline{\mathbf{R}}_{M, N}(\xi, t ; \wp)=\Psi_{M}^{T}(t)\left(-\overline{\mathbf{G}}(\wp)-\underline{\mathbf{K}}(\wp)+\mathbf{P}^{(v)^{T}} \underline{\mathbf{K}}(\wp) \mathbf{D}^{(\lambda)}-\mathbf{P}^{(v)^{T}} \underline{\mathbf{F}}(\wp)\right) \Psi_{N}(\xi)
$$

for upper function, the approximate solution $\varphi_{M, N}(\xi, t ; \wp)$ for $F C g H F_{P}^{(i i)}$-differentiability is obtained.

\section{Illustrative Examples}

In this sequel, we exemplify shifted Chebyshev spectral-tau method on some examples of fuzzy fractional diffusion equation, fuzzy fractional heat equation and fuzzy fractional wave equation. Each equation is converted to two different systems according to the $F C g H F_{P}$-differentiability of the fuzzy-valued function. Graphical solutions are obtained using Mathematica 10.

\section{Example 6.1}

Consider the following fuzzy fractional diffusion equation

$$
{ }_{g H}^{C} \mathscr{D}_{t}^{\nu} \varphi(\xi, t) \ominus_{g H}^{C} \mathscr{D}_{\xi}^{\lambda} \varphi(\xi, t)=\varphi(\xi, t)
$$

for $0<\xi \leq 1,0<t \leq 1,0<\nu \leq 1$ and $0<\lambda \leq 2$, subjected to boundary conditions 


$$
\varphi(\xi, 0)=[\wp-1,1-\wp] \xi^{3}, \quad \varphi(0, t)=0, \quad \varphi(1, t)=0
$$

on integrating we obtain the following equations, for $\wp \in[0,1]$

$$
\varphi(\xi, t ; \wp) \ominus[\wp-1,1-\wp] \xi^{3} \ominus \mathscr{I}_{t}^{\nu C}{ }_{g H} \mathscr{D}_{\xi}^{\lambda} \varphi(\xi, t ; \wp)=\mathscr{I}_{t}^{\nu} \varphi(\xi, t ; \wp)
$$

and

$$
-[\wp-1,1-\wp] \xi^{3} \ominus(-\varphi(\xi, t ; \wp)) \oplus\left(-\mathscr{I}_{t}^{\nu C} \mathscr{D}_{\xi}^{\lambda} \varphi(\xi, t ; \wp)\right)=\ominus\left(-\mathscr{I}_{t}^{\nu} \varphi(\xi, t ; \wp)\right)
$$

Subsequent to the approximations and methodology defined in Section 5.1, we obtain the approximate solution from 25 fuzzy linear algebraic equations generated by taking $N=4$ and $M=4$. Fig. 1 plots the solutions of $\varphi(\xi, t)$ by using Eq. (80) for $\nu=0.85$ and $\lambda=1.95$, whereas for Eq. (81) same graphical view is found for $\nu=0.85$ and $\lambda=1.95$. Fig. 2 shows the point wise error variation between exact values and calculated values of $\varphi(\xi, t)$.

\section{Example 6.2}

Consider the following fuzzy fractional heat equation

$$
{ }_{g H}^{C} \mathscr{D}_{t}^{\nu} \varphi(\xi, t) \ominus 4 \odot{ }_{g H}^{C} \mathscr{D}_{\xi}^{\lambda} \varphi(\xi, t)=0
$$

for $0<\xi \leq 1,0<t \leq 1,0<\nu \leq 1,0<\lambda \leq 2$, subjected to boundary conditions

$$
\varphi(\xi, 0)=[\wp-1,1-\wp] \sin (\pi \xi), \quad \varphi(0, t)=0, \quad \varphi(1, t)=0
$$

on integrating we obtain the following equations

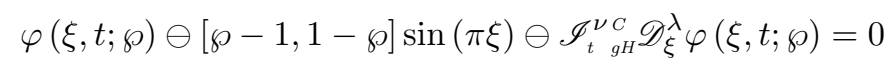

and

$$
-[\wp-1,1-\wp] \sin (\pi \xi) \ominus(-\varphi(\xi, t ; \wp)) \oplus\left(-\mathscr{I}_{t}^{\nu C} \mathscr{g}_{g H}^{\lambda} \varphi(\xi, t ; \wp)\right)=0
$$

Following the approximations and scheme defined in Section 5.1, and taking $N=4$ and $M=4,25$ fuzzy linear algebraic equations are generated and solution of $\varphi(\xi, t)$ is obtained. Fig.3 draws the solution of $\varphi(\xi, t)$ on using Eq. (84), for $\nu=0.95$ and $\lambda=1.025$ and same result is for Eq. (85). The point wise error variation between exact values and calculated values of $\varphi(\xi, t)$ is shown in Fig.4.

\section{Example 6.3}


Consider the following fuzzy fractional wave equation

$$
{ }_{g H}^{C} \mathscr{D}_{t}^{\nu} \varphi(\xi, t) \oplus_{g H}^{C} \mathscr{D}_{\xi}^{\lambda} \varphi(\xi, t)=[\wp-1,1-\wp] \xi e^{t}
$$

for $0<\xi \leq 2,0<t \leq 1,0<\nu \leq 2,0<\lambda \leq 2$, subjected to boundary conditions

$$
\varphi(\xi, 0)=[\wp-1,1-\wp] \xi, \quad \partial_{t} \varphi(\xi, 0)=0, \quad \varphi(0, t)=0, \quad \varphi(2, t)=[\wp-1,1-\wp] 2 e^{t}
$$

on integrating we obtain the following equations

$$
\varphi(\xi, t ; \wp) \ominus[\wp-1,1-\wp] \xi \oplus \mathscr{I}_{t}^{\nu C} \mathscr{D}_{\xi}^{\lambda} \varphi(\xi, t ; \wp)=\mathscr{I}_{t}^{\nu}[\wp-1,1-\wp] \xi e^{t}
$$

and

$$
-[\wp-1,1-\wp] \xi \ominus(-\varphi(\xi, t ; \wp)) \ominus\left(-\mathscr{I}_{t g h}^{\nu C} \mathscr{D}_{\xi}^{\lambda} \varphi(\xi, t ; \wp)\right)=\ominus\left(-\mathscr{I}_{t}^{\nu}[\wp-1,1-\wp] \xi e^{t}\right)
$$

Subsequent to the approximations and scheme defined in Section 5.2, we attain the solutions of $\varphi(\xi, t)$ by solving 25 fuzzy linear algebraic equations that are generated by taking $N=4$ and $M=4$. Solutions of Eqs.(88) and (89) for $\nu=1.95$ and $\lambda=1.95$ are plotted in Figs.5 and 6, respectively. Figs.7 and 8 depict the point wise error variation between exact values and calculated values of $\varphi(\xi, t)$ obtained from Eqs.(88) and (89), accordingly.

\section{Conclusions}

In this manuscript, we concerned with the fuzzy fractional diffusion equation and fuzzy fractional wave equation in the direction of time and space both. The basic descriptions of fuzzy calculus for one and two dimensional fuzzy-valued functions were elaborated. Moreover, the fuzzy Caputo gH-partially-differentiability of increasing and decreasing bivariate fuzzy-valued functions, on the basis of which each example of FFDE and FFWE were converted into two equations, had been discussed. Solutions of each example were shown graphically for different values of $\nu$ and $\lambda$. Error bar plots were depicted in comparison with exact numerical solutions that had been obtained by using an appropriate command on Mathematica 10. Consequently, from facts and figures obtained from Section 6 , we have the following conclusions

- Spectral-tau method was found to be highly applicable to obtain effective fuzzy solutions of FFDEs and FFWEs.

- This method completely discretizes the integral and differential equations that simplifies the problem to a great extent and calculations of derivatives and integrals at different orders just becomes the matter of changing the values of $\nu$ and $\lambda$ in the analytical form of SCOM of derivative and integration. 
- Small number of $M$ and $N$ is required to generate accurate fuzzy solutions of FFDEs and FFWEs, subjected to initial and boundary conditions, graphically.

- Error analysis through error bar plots is significantly advantageous to cope out the point wise errors as it locates the point wise variation between the exact and calculated values.

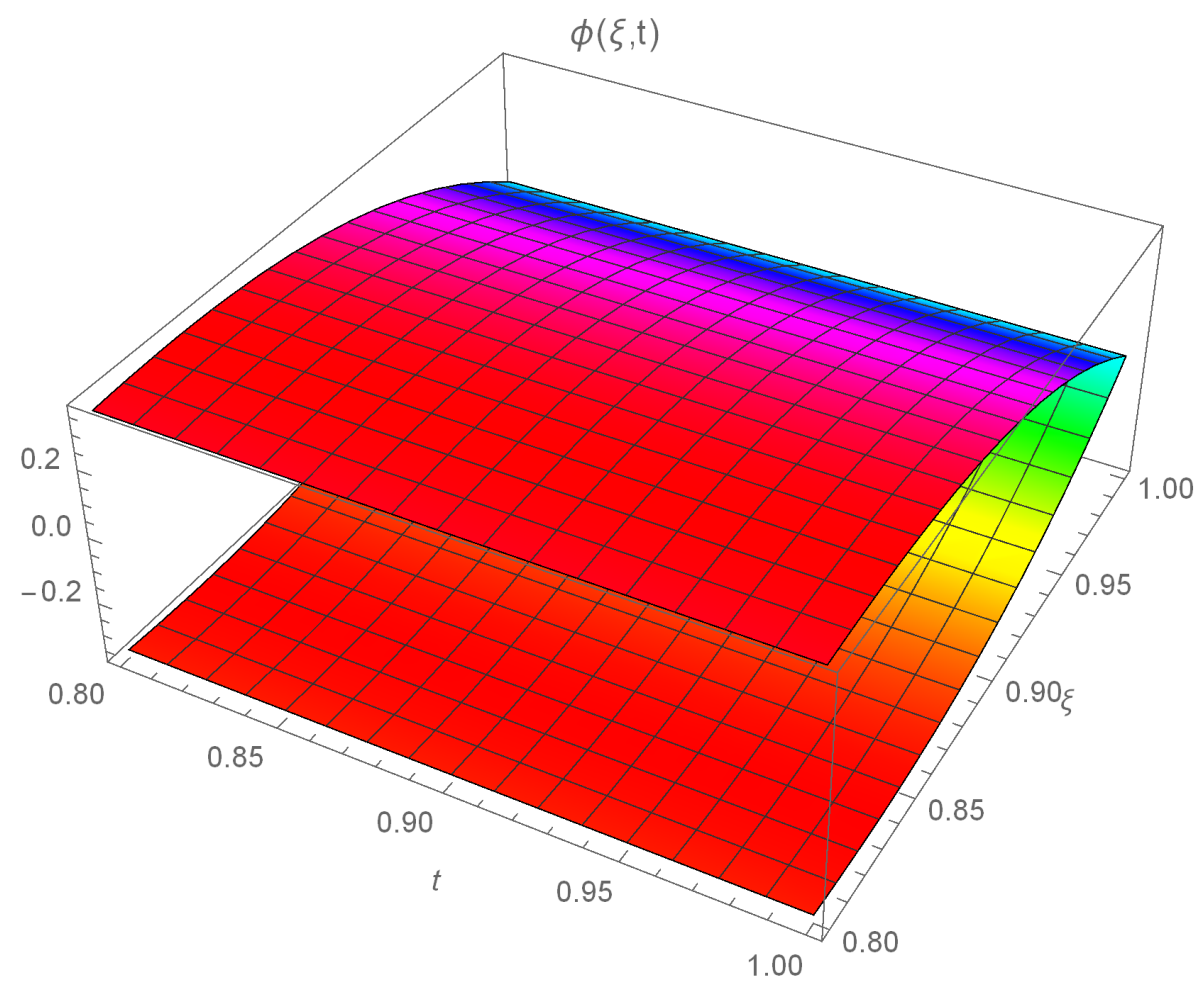

Figure 1: Fuzzy solutions of Example 6.1 for $\wp=0.6$ 


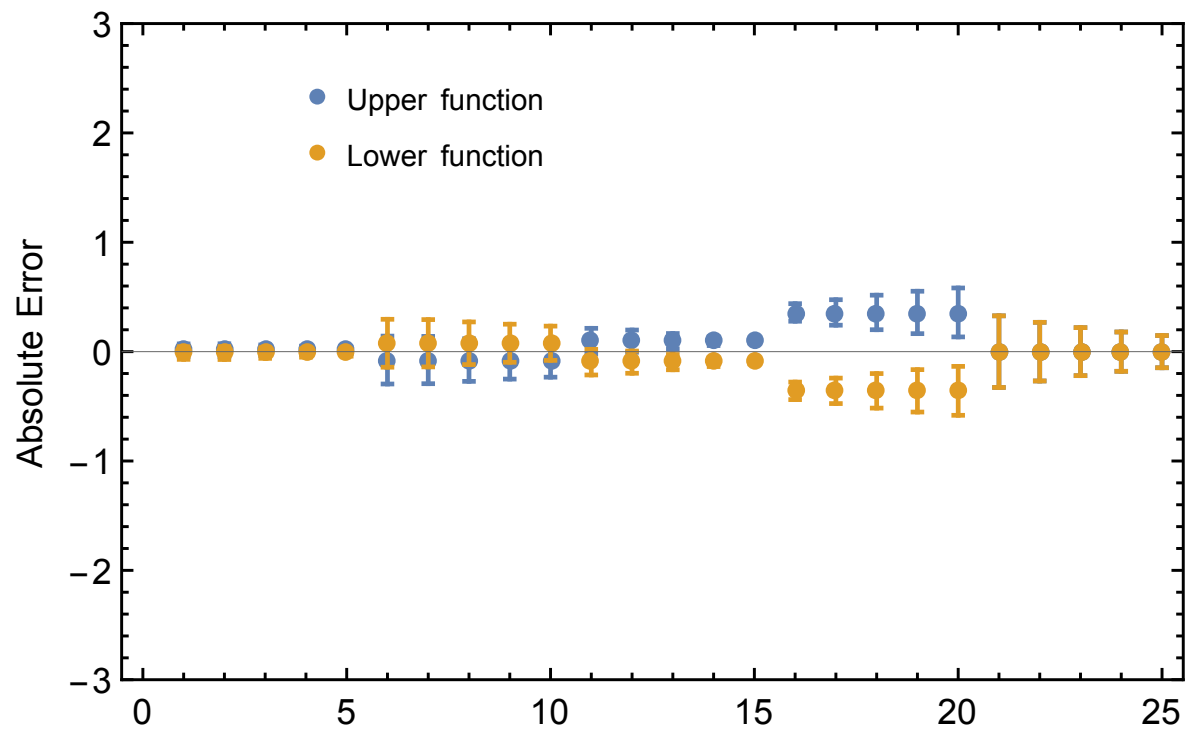

Figure 2: Error bar plots of 25 points of calculated and exact values Example 6.1 


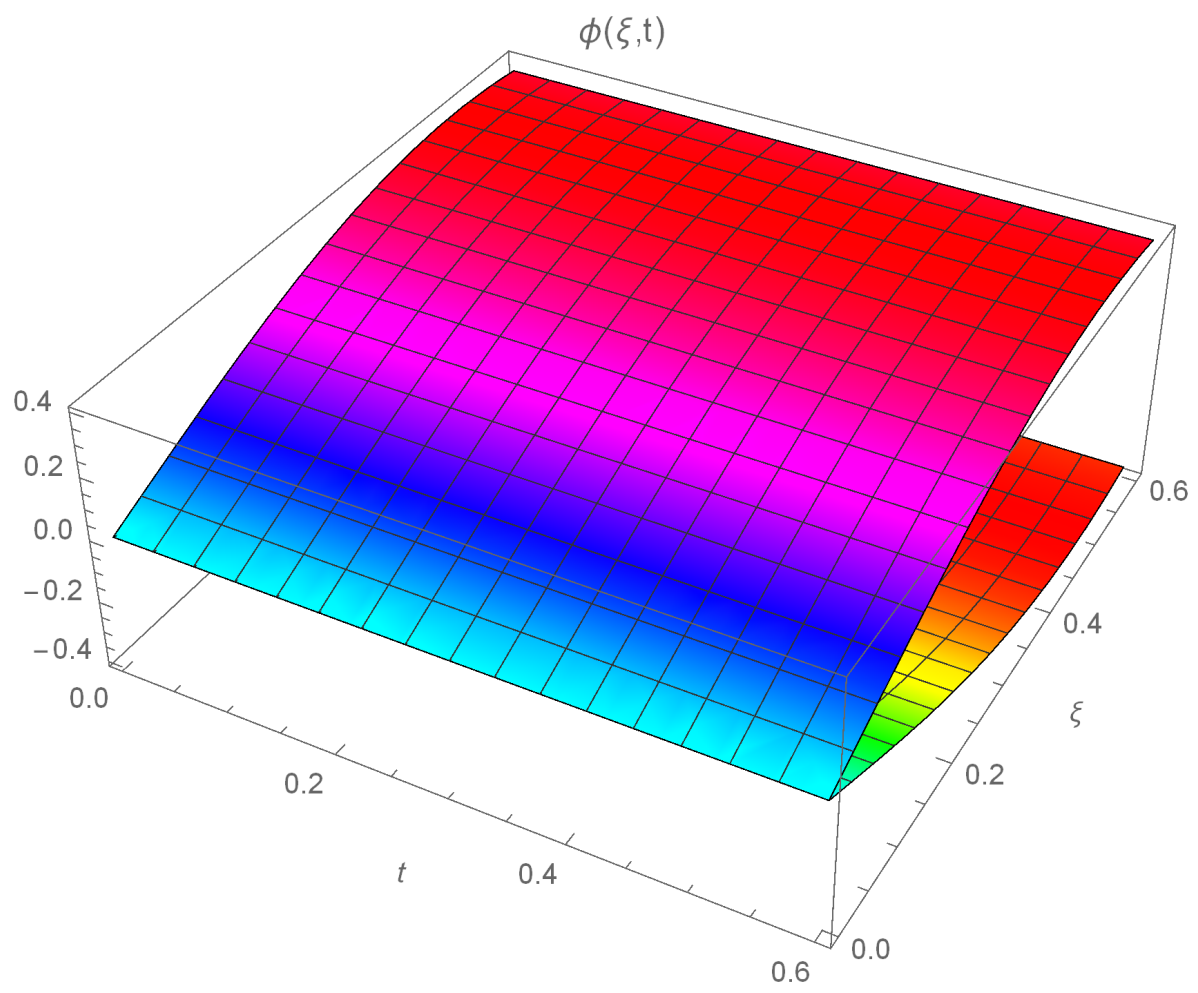

Figure 3: Fuzzy solutions of Example 6.2 for $\wp=0.6$ 


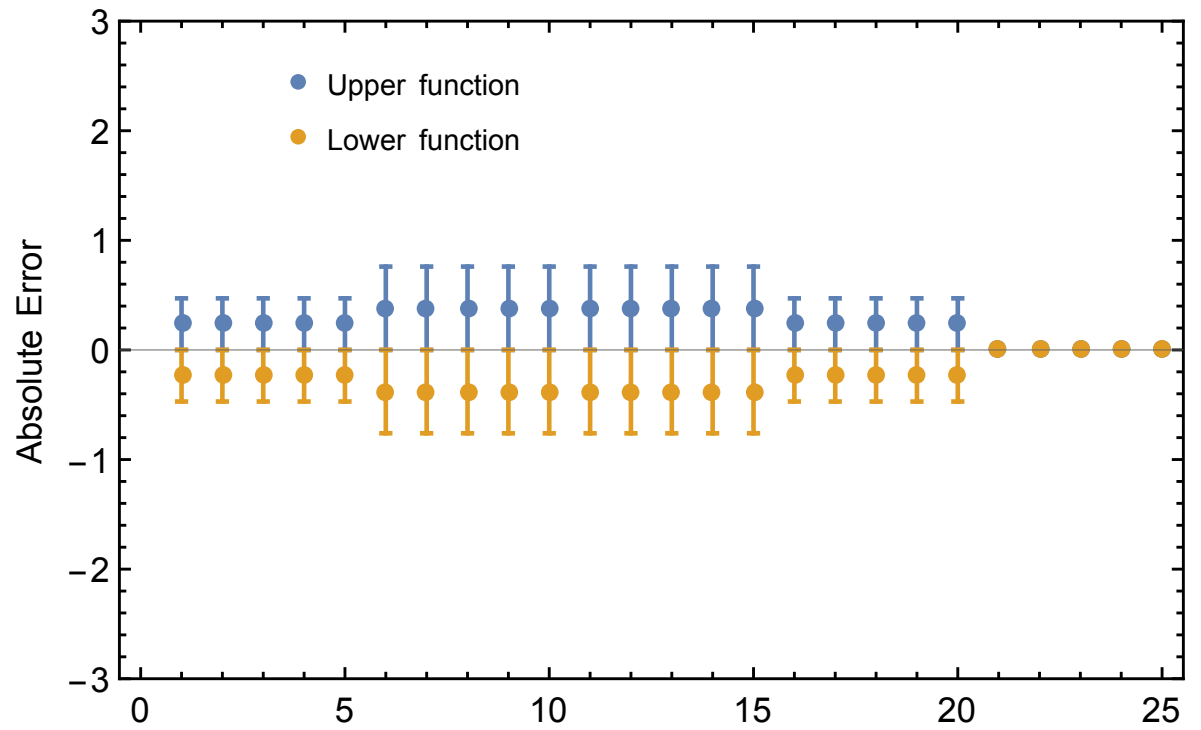

Figure 4: Error bar plots of 25 points of calculated and exact values Example 6.2 


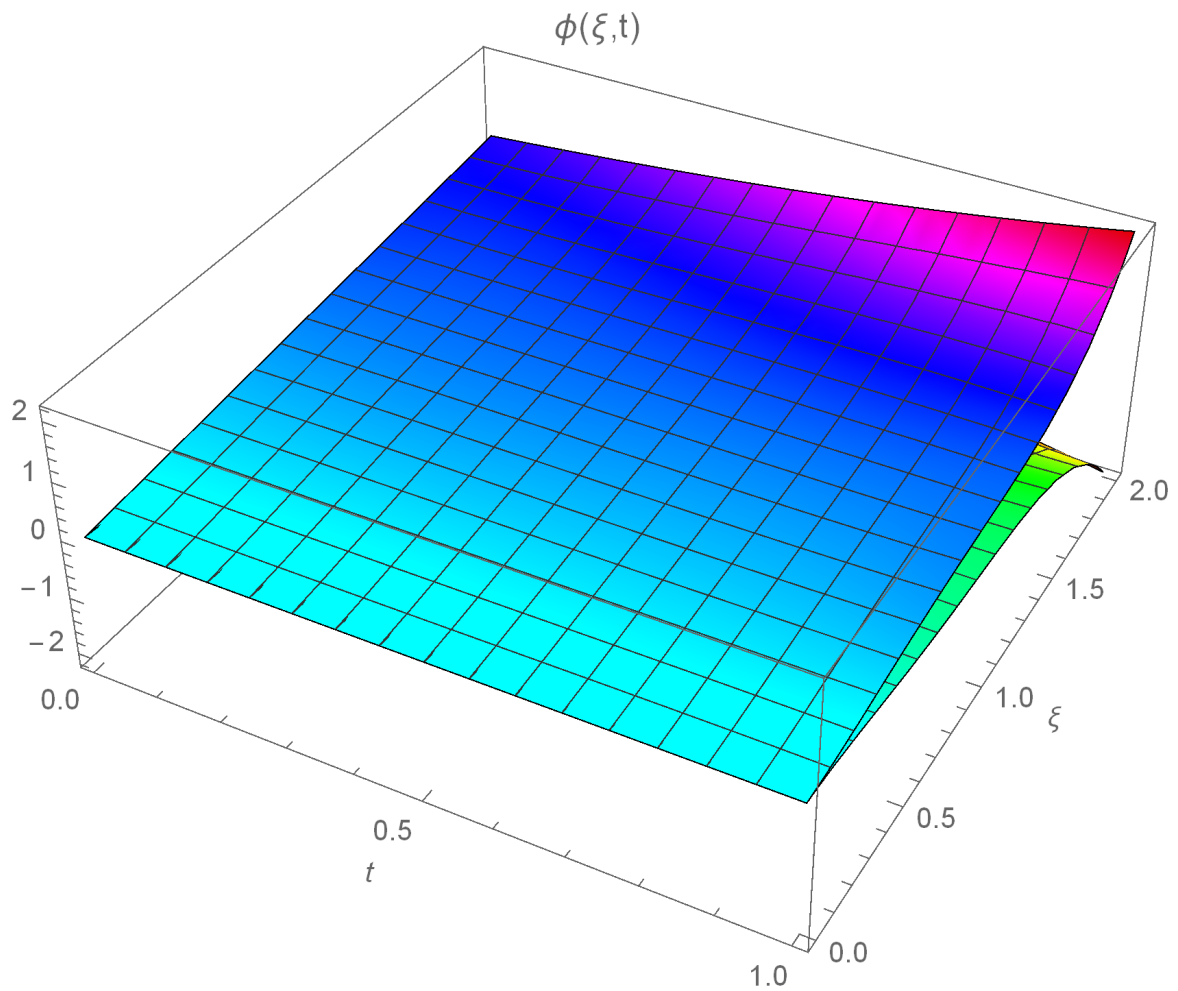

Figure 5: Fuzzy solutions of Example 6.3 for $\wp=0.6$ 


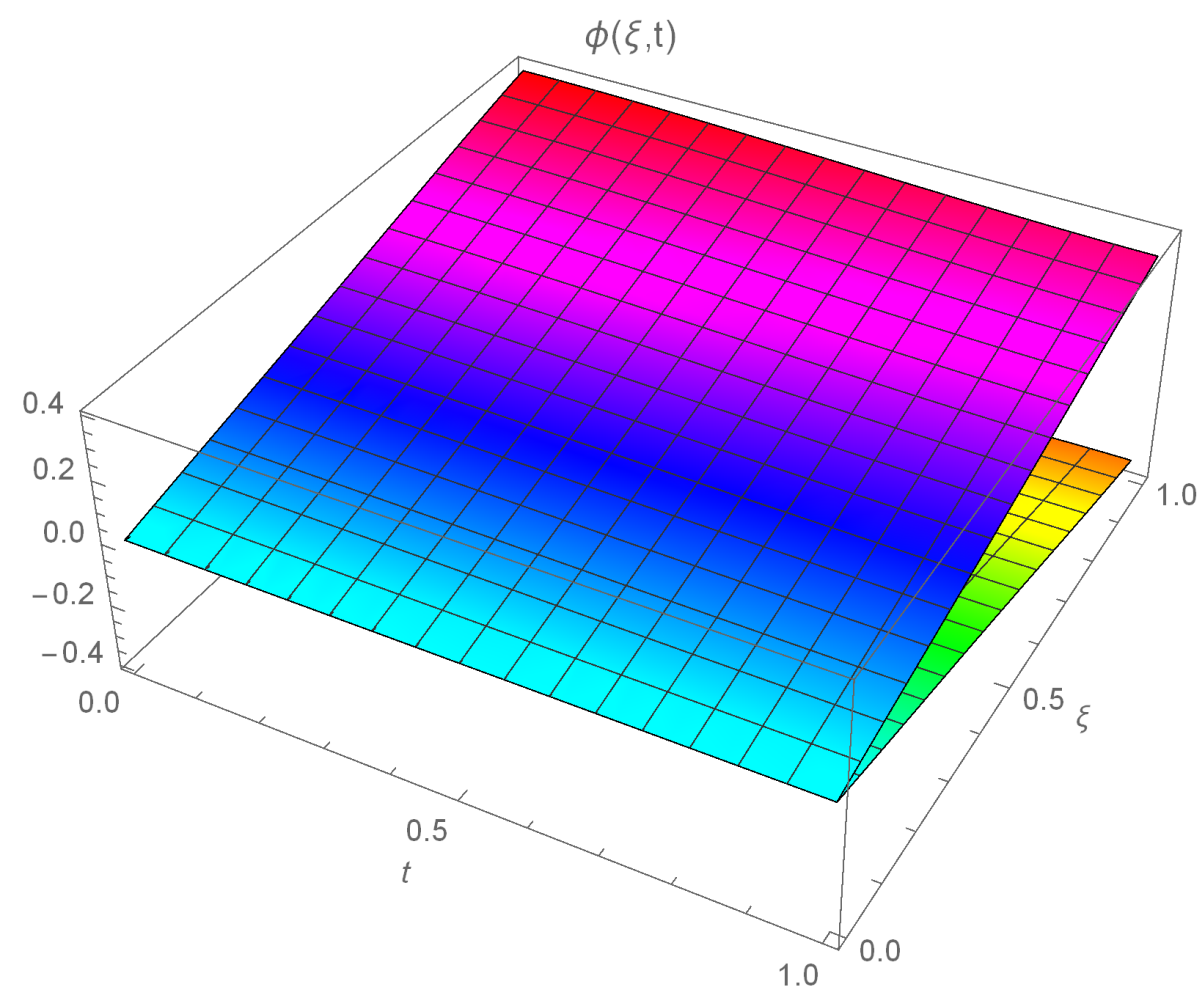

Figure 6: Fuzzy solutions of Example 6.3 for $\wp=0.6$ 


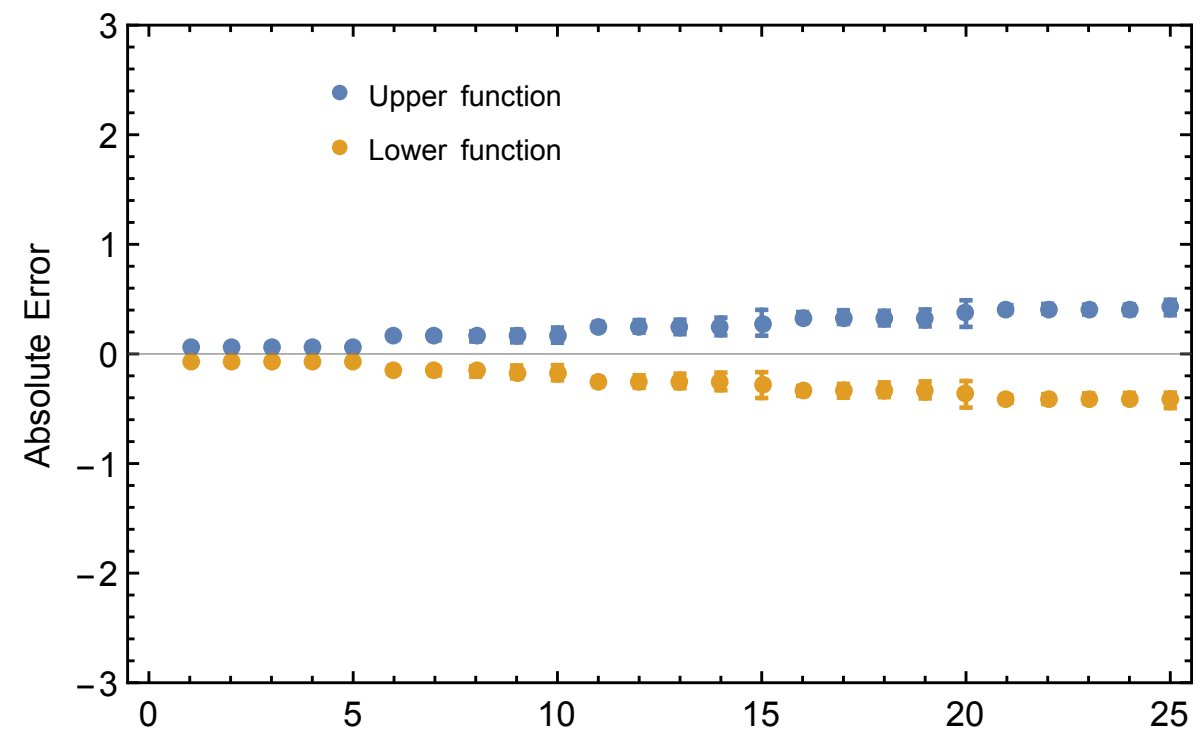

Figure 7: Error bar plots of 25 points of calculated and exact values Example 6.3 using Eq.(89) 


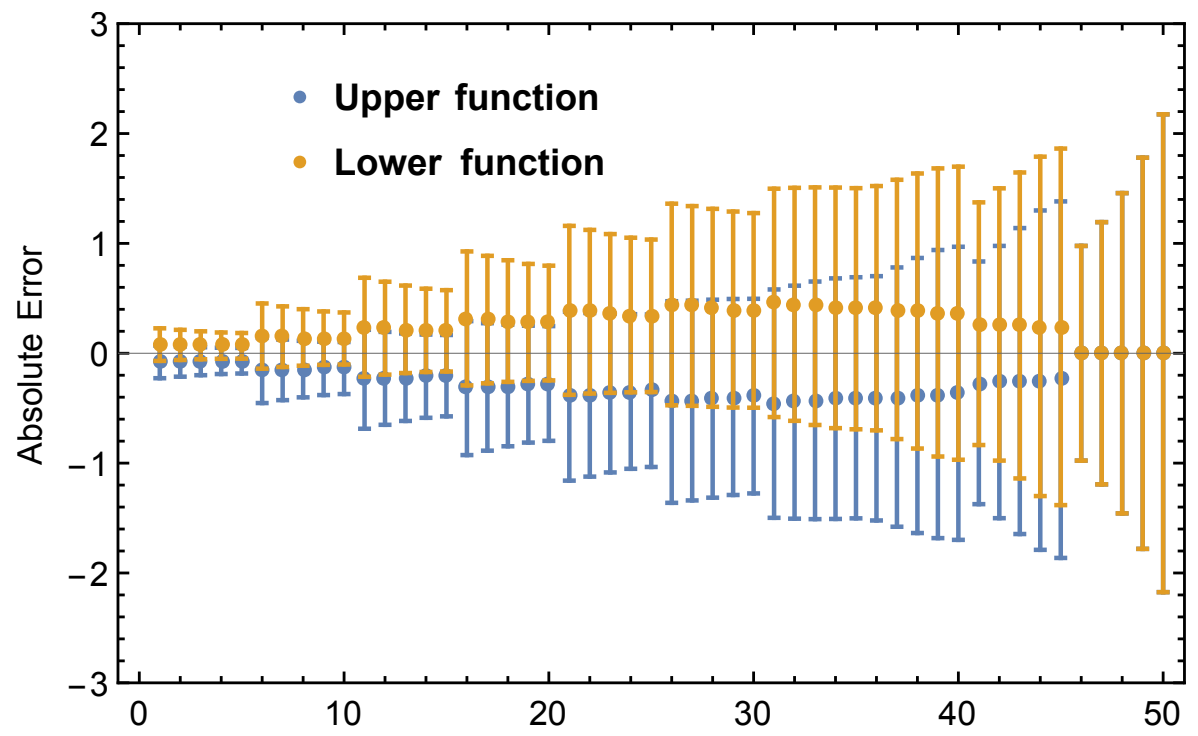

Figure 8: Error bar plots of 25 points of calculated and exact values Example 6.3 using Eq.(89)

\section{References}

[1] N.A. Khan, F. Riaz and O.A. Razzaq, A comparison between numerical methods for solving fuzzy fractional differential equations, Nonlinear Eng. 3(3) (2014), 155-162.

[2] A. Khastan, I. Perfilieva and Z. Alijani, A new fuzzy approximation method to Cauchy problems by fuzzy transform, Fuzzy Sets Sys. (2015), dx.doi.org/10.1016/j.fss.2015.01.001

[3] N.A. Khan, O.A. Razzaq and M. Ayyaz, On the solution of fuzzy differential equations by Fuzzy Sumudu Transform, Nonlinear Eng. 4(1) (2015), 49-60.

[4] T. Allahviranloo, S. Abbasbandy and S.S. Behzadi, Solving nonlinear fuzzy differential equations by using fuzzy variational iteration method, Soft Comput. 18(11) (2015), 2191-2200.

[5] J.J. Buckley and T. Feuring, Introduction to fuzzy partial differential equations, Fuzzy Sets Sys. 105 (1999), 241-248.

[6] S.S. Behzadi, Solving Cauchy reaction-diffusion equation by using Picard method, Springer Plus 2(108) (2013), doi = 10.1186/2193-1801-2-108.

[7] B. Ghazanfari and P. Ebrahimi, Differential transformation method for solving fuzzy fractional heat equations, Int. J. Math. Model. Comput. 5(1) (2015), 81-89.

[8] T. Allahvirnaloo and M.A Kermani, Numerical methods for fuzzy linear partial differential equations under new definition of derivative, Iranian J. Fuzzy Sys. 7(3) (2010), 33-50. 
[9] T. Allahvirnaloo, Z. Gouyandeh, A. Armand and A. Hasanoglu, On fuzzy solutions for heat equation based on generalized Hukuhara differentiability, Fuzzy Sets Sys. 256 (2015), 1-23.

[10] T. Allahvirnaloo, S. Abbasbandy and H. Rouhparvar, The exact solutions of fuzzy wave-like equations with variable coefficients by a variational iteration method, App. Soft. Comput. 11 (2011), 2186-2192.

[11] A.H. Bhrawy and M.M. Shomrani, A shifted Legendre spectral method for fractional-order multi-point boundary value problems, Adv. Diff. Eq. 2012(8) (2012).

[12] A.H. Bhrawy and M. A. Alghamdi, A Legendre tau-spectral method for solving timefractional heat equation with nonlocal conditions, The Sci. World J. 2014(7) (2104), doi = dx.doi.org/10.1155/2014/706296.

[13] A. Ahmadian, M. Suleiman and S. Salahshour, An operational matrix based on Legendre polynomials for solving fuzzy fractional-order differential equations, Abs. App. Anal. 2013 (2013), doi $=$ dx.doi.org/10.1155/2013/505903

[14] A. Ahmadian, S. Salahshour, D. Baleanu, H. Amirkhani and R. Yunus, Tau method for the numerical solution of a fuzzy fractional kinetic model and its application to the oil palm frond as a promising source of xylose, J. Comput. Phys. (2015), 1-27.

[15] E.H. Doha, A.H. Bhrawy and S.S. Ezz-Eldien, Efficient Chebyshev spectral methods for solving multi-term fractional orders differential equations, Appl. Math. Model. Simul. Comput. Eng. Enviro. Sys. 35(12) (2011), 5662-5672.

[16] R. Ren, H. Li, W. Jiang and M. Song, An efficient Chebyshev-tau method for solving the space fractional diffusion equations, Appl. Math. Comput. 224 (2013), 259-267.

[17] W. Bao and J. Shen, A generalized-Laguerre-Hermite pseudospectral method for computing symmetric and central vortex states in Bose-Einstein condensates, J. Comput. Phys. 227 (2008), 9778-9793.

[18] B.Y. Guo, J. Shen and C.L. Xu, Spectral and pseudospectral approximations using Hermite functions: application to the Dirac equation, Adv. Comput. Math. 19 (2003), 35-55.

[19] D. Baleanu, A.H. Bhrawy and T.M. Taha, Two efficient generalized Laguerre spectral algorithms for fractional initial value problems, Abs. Appl. Anal. 2013 (2013), doi = dx.doi.org/10.1155/2013/546502.

[20] A.H. Bhrawy, Y.A. Alhamed, D. Baleanu and A.A. Al-Zahrani, New spectral techniques for systems of fractional differential equations using fractional-order generalized Laguerre orthogonal functions, Fract. Calc. Appl. Anal. 17 (2014) 1137-1157.

[21] E.H. Doha, A.H Bhrawy and S.S. Ezz-Eldien, A new Jacobi operational matrix: an application for solving fractional diffusion equations, Appl. Math. Model. 36(10) (2012), 4931-4943.

[22] E.H. Doha, A.H Bhrawy and S.S. Ezz-Eldien, A Chebyshev spectral method based on operational matrix for initial and boundary value problems of fractional order, Comp. Math. Appl. 62(5) (2011), 2364-2373. 
[23] A.H. Bhrawy and A.S. Alofi, The operational matrix of fractional integration for shifted Chebyshev polynomials, Appl. Math. Letter. 26 (2013), 25-31.

[24] A.H. Bhrawy, M.M. Alghamdi and T.M. Taha, A new modified generalized Laguerre operational matrix of fractional integration for solving fractional differential equations on the half line, Adv. Diff. Eq. 0(179) (2012).

[25] S. Hajighasemi and S.M. Khorasani, Numerical solution of algebraic fuzzy equations by Adomian method, Appl. Math. Sci. 4(71) (2010), 3509-3514.

[26] L.A. Zadeh, Fuzzy sets as a basis for a theory of possibility, Fuzzy Sets Sys. 1(1) (1978), 3-28.

[27] V.H. Ngo, Fuzzy fractional functional integral and differential equations, Fuzzy Sets Sys. 280 (2015), 58-90.

[28] B. Bede and L. Stefanini, Generalized differentiability of fuzzy-valued functions, Fuzzy Sets Sys. 230 (2013), 119-141. 